\title{
A MACHINE LEARNING MODEL TO PREDICT SEIZURE SUSCEPTIBILITY FROM RESTING-STATE FMRI CONNECTIVITY
}

\author{
Rachael Garner \\ Marianna La Rocca \\ Giuseppe Barisano \\ Arthur W. Toga \\ Dominique Duncan
}

\author{
Paul Vespa \\ Department of Neurosurgery \\ University of California, Los Angeles \\ 300 Stein Plaza \\ Suite 420 \\ Los Angeles, CA, USA \\ pvespa@mednet.ucla.edu
}

\begin{abstract}
Traumatic brain injury (TBI) is a leading cause of disability globally. Many patients develop post-traumatic epilepsy, or recurrent seizures following TBI. In recent years, significant efforts have been made to identify biomarkers of epileptogenesis that may assist in preventing seizure occurrence by identifying high-risk patients. We present a novel method of assessing seizure susceptibility using data from 49 patients enrolled in the Epilepsy Bioinformatics Study for Antiepileptogenic Therapy (EpiBioS4Rx). We employ a machine learning paradigm that utilizes a Random Forest classifier trained with resting-state functional magnetic resonance imaging (fMRI) data to predict seizure outcomes. Following 100 rounds of stratified crossvalidation with $70 \%$ of resting state fMRI scans as the training set and $30 \%$ as the testing set, our model was found to assess seizure outcome in the testing set with $69 \%$ accuracy. To validate the method, we compared our results with classification by Support Vector Machines and Neural Network classifiers.
\end{abstract}

Keywords: traumatic brain injury, post-traumatic epilepsy, seizure prediction, machine learning, fMRI

\section{INTRODUCTION}

An estimated 10 million individuals experience a traumatic brain injury (TBI) each year, contributing to the World Health Organization's classification of TBI as the third largest contributor to death and disability globally (Humphreys et al. 2013). One common sequelae of TBI that causes significant disability within patient populations is post-traumatic epilepsy (PTE), or recurrent seizures following brain injury. In recent years, widespread efforts have been undertaken to examine the mechanisms of epileptogenesis following TBI to identify and potentially reduce seizure susceptibility, although at this time, the progression of PTE is still not well understood.

Efficient, large-scale trials of prophylactic anticonvulsant therapies are challenging within the PTE population, as many TBI patients will never develop seizures, and those who develop PTE may experience 
their first seizure more than 10 years after injury (Frey 2003). In an effort to enrich patient populations for potential antiepileptogenic therapy trials, as well as to predict PTE development for a given individual, biomarkers of epileptogenesis following injury have been explored. Several reviews have elaborated on electrophysiological and imaging biomarkers of epileptogenesis following injury (Mishra et al. 2011; Immonen et al. 2013; Staba, Stead, and Worrell 2014).

In this study, we examine the potential role that functional magnetic resonance imaging (fMRI) may have as a noninvasive biomarker of seizure susceptibility following TBI. fMRI allows investigators to examine patterns of neural network activation, both in clinical populations and experimental models. Several studies have reported that hyperconnectivity following TBI is associated with positive brain plasticity and longterm outcome in patient populations (Iraji et al. 2016; F. G. Hillary et al. 2011; Frank G. Hillary et al. 2014). A recent study also shows that resting state fMRI may indicate persistent alterations in functional network connectivity following experimentally induced TBI in rats (Mishra et al. 2014), and in human epileptic patients (Rajpoot et al. 2015; Zhang et al. 2012). As fMRI has shown to be useful in identifying functional alterations and recovery coinciding with brain injury and epilepsy, it is potentially useful in identifying epileptogenesis following injury.

In this study, we hope to explore how network alterations in human TBI patients may influence the development of PTE. We utilize fMRI to examine how resting-state blood oxygen level dependent (BOLD) connectivity may differ between TBI patients that go on to present with seizures and seizure-free patients.

In recent years, several studies have demonstrated that machine learning models, specifically Support Vector Machines (SVM), deep learning networks, and Random Forest (RF) classifiers, may successfully predict seizures from scalp encephalography (EEG) signals and intracranial EEG. On average, SVM models predicted seizures 22.5 minutes before seizure onset with sensitivity ranging from $71.97 \%-73.9 \%$ (Rasekhi et al. 2013; Teixeira et al. 2014; Bandarabadi et al. 2015; Usman, Usman, and Fong 2017). A deep learning algorithm integrated with IBM's TrueNorth processor predicted seizures up to 48 hours prior to onset with a sensitivity of $69 \%$ (Kiral-Kornek et al. 2018). Seizure detection utilizing the RF classifier has shown the most promising results, reaching an average sensitivity of $98.5 \%$ when combined with SVM (Mursalin et al. 2017) and $99.29 \%$ when EEG was mapped into a one-dimensional space prior to learning (PintoOrellana and Cerqueira 2017).

However, these methods have investigated patient populations already diagnosed with epilepsy, so the machine learning tools serve to predict seizure-onset in real-time, rather than predict an epileptic condition. In an effort to establish an automated method to detect epilepsy in its early stages, Fergus et al. built a kNearest Neighbors machine learning algorithm that classifies seizure and non-seizure events from scalp EEG recordings, obtaining $88 \%$ specificity and sensitivity (Fergus et al. 2016).

Several studies have reported improved results by combining machine learning techniques with complex preprocessing tools and other algorithms. Such studies have classified epileptic versus non-epileptic controls from EEG data utilizing a tensor-based scheme (Pippa et al. 2017) and a k-Nearest Neighbors algorithm (Karlık and Hayta 2014) reaching accuracies of $97.7 \%$ and $99.75 \%$ respectfully.

Two studies have utilized machine learning algorithms to predict outcomes in TBI patients. Obukhov et al. used pattern detection in EEG to extract key electrophysiological phenomena associated with epileptiform activity, ultimately predicting PTE in rats with $80 \%$ accuracy (Obukhov et al. 2018). Messosi et al. have performed a PTE prediction study using human MRI, but obtained only statistical evaluations of PTE risk factors (Messori A. et al. 2005). At the time of writing, only one study could be found that used machine learning models to classify epileptic patients based on fMRI data, reporting that functional connectivity (FC) was an effective training dataset (sensitivity $=85 \%$ ) to identify epileptic patients versus healthy controls in a pattern classification model (Zhang et al. 2012).

Although such models may be useful in identifying the neuromarkers of an existing epileptic condition or predicting seizure onset, they have not been used to predict seizure susceptibility in a patient-only 
population. In this study, we explore the predictive power of resting-state fMRI using several established machine learning paradigms. First, RF is used to extract important features from correlation coefficient matrices of FC. Then we compare how well RF, SVM, and Neural Network (NN) models classify subjects that report seizures following injury and seizure-free subjects to explore the feasibility of fMRI as a biomarker of seizure susceptibility.

\section{METHODS}

\subsection{Subjects}

Patient scans and clinical data were acquired from the Epilepsy Bioinformatics Study for Antiepileptogenic Therapy (EpiBioS4Rx). Study inclusion criteria were: age between 6 and 100, moderate to severe acute traumatic injury, Glasgow Coma Score of 3-12 recorded after injury without continuous sedation at time of enrollment, and hemorrhagic contusional injury to frontal and/or temporal lobes. Exclusion criteria included: diffuse axonal injury in the absence of hemorrhagic contusions or skull fracture, TBI defined by isolated epidural hemorrhage that improved after evacuation, isolated anoxic brain injury, devastating cervical spine injury, and pre-existing neurological disorders, seizures, and dementia. A total of 49 subjects had initial fMRI scans and clinical seizure data available for analysis. We classified 11 patients as the seizure group, as each experienced at least one seizure within 6 months of injury.

The subjects (average age $=43, \max =85, \min =8$ ) or a legal authorized representative gave written informed consent to participate in the study. The study sample includes patients enrolled through Massachusetts General Hospital, Phoenix Children's Hospital, University of California Davis, University of California Los Angeles, University of Cincinnati, University of Miami, Yale University, and Alfred Hospital.

The EpiBioS4Rx protocol was designed to gather patient data, but not healthy controls. Although healthy subjects may be acquired from other publicly available cohorts to expand the training set of our classifiers, we chose to select only patients within the EpiBioS4Rx cohort to assess the core objective of the study: can epilepsy be predicted following TBI? Thus, our subject population is comprised of only TBI subjects and no healthy controls.

\subsection{Clinical Data}

Anonymized clinical data were obtained from REDCap. Enrollment in the study required the completion of several questionnaires and surveys. Basic patient demographic information (including date of birth and date of injury) were extracted from a patient background information instrument. Data related to incidence of seizures were extracted from the PTE Ottman Questionnaire and Glasgow Outcome Scale-Extended (GOSE), which were administered to subjects 30, 90, and 180 days post-injury. In the survey, subjects were asked if they had any seizures after his or her brain injury. Because the questionnaire was administered several times, we could assess an estimation of the latency prior to seizure onset for some patients that had complete datasets available (i.e. no seizures prior to 90 days following injury, but seizure onset began between 90-180 days post-injury). As not all subjects had complete forms available, we elected to classify patients within binary seizure or non-seizure groups. Each subject in the seizure group had at least 1 seizure within 6 months of injury.

\section{3 fMRI Acquisition}

Each subject underwent resting-state fMRI scanning within 23 days injury (average $=12.7$ ). Resting-state connectivity was collected at eight clinical sites, following a standardized protocol (Table 1). All scans were performed with 3T MRI, although the scanner model differed by institution. 
Table 1: The BOLD-fMRI protocol used in EpiBioS4Rx to assess resting-state connectivity.

\begin{tabular}{|l|l|l|l|l|l|l|}
\hline Sequence & TR $[\mathrm{ms}]$ & TE $[\mathrm{ms}]$ & FA [deg] & $\begin{array}{l}\text { Thickness } \\
{[\mathrm{mm}]}\end{array}$ & FOV [\%] & Matrix \\
\hline $\begin{array}{l}\text { EpiBioS4Rx } \\
\text { BOLD fMRI }\end{array}$ & 2000 & 30 & 78 & 3.0 & 100 & $64 \times 64$ \\
\hline
\end{tabular}

\section{4 fMRI Analysis}

Raw DICOM images were downloaded from the Image and Data Archive and preprocessed using the Data Processing Assistant for Resting-State fMRI (DPARSF) toolbox (Yan 2010), based on Statistical Parametric Mapping (SPM12) (Friston et al. 1994). Standard preprocessing of functional images included the following steps:

\section{Conversion from DICOM to NIFTI format}

2. Removal of first 10 time points to account for subject acclimation to scanner

3. Slice-time correction and realignment of remaining volume

4. Spatial normalization to the Montreal Neurological Institute (MNI) EPI template with resampling voxel side of $3 \mathrm{~mm} \times 3 \mathrm{~mm} \times 3 \mathrm{~mm}$

5. Spatial smoothing with Gaussian filter with full-width at half maximum and kernel size $4 \mathrm{~mm} x$ $4 \mathrm{~mm} \times 4 \mathrm{~mm}$

6. Detrending and removal of nuisance covariates (white matter and cerebrospinal fluid signals as well as head motion) using linear regression with a polynomial trend of 2

7. Finally, signals between 0.01 and $0.08 \mathrm{~Hz}$ were filtered out.

Following data preprocessing, 116 regions of interest (ROIs) were defined based on the Automated Anatomical Labeling (AAL) atlas. FC matrices were extracted for connectivity between all combinations of the ROIs. FC correlation coefficients were then computed to build a 116x116 matrix to be used in the machine learning paradigm.

\subsection{Machine learning model}

FC correlation coefficients, totaling 6670 per subject, were first used as features to train a RF classifier and discriminate TBI patients that have experienced at least 1 seizure from seizure-free patients. Then, to validate the results from RF classification, we assessed seizure susceptibility following TBI using two other models: SVM and NN classifiers. The RF classifier was initially chosen for its robustness, proven effectiveness in predicting prior seizure onset (Mursalin et al. 2017; Pinto-Orellana and Cerqueira 2017), and success in early detection of Alzheimer's disease (Amoroso et al. 2018).

Random Forests are an ensemble of classification trees, whose trees are grown by bootstrapping training data and randomly selecting a candidate set of features at each branching point. At each split the square root of the feature number is randomly picked and each tree is grown unpruned to obtain low-bias models. $\mathrm{RF}$ is a robust model that does not overfit thanks to internal bagging, and it provides a continuous measure of feature importance (Breiman 2001). We performed 100 rounds of stratified cross-validation, and for each round, classification performances were measured in term of accuracy, sensitivity and specificity using $70 \%$ of the subjects as the training set and the other $30 \%$ as the testing set. We performed a feature selection nested within each cross-validation round. For each round we selected and recorded features exceeding the third quartile of the importance distribution computed in terms of mean accuracy decrease. Then, we used a hypothesis test to evaluate which feature's occurrences had not happened by chance over the 100 rounds 
and selected them as the final important features. The feature selection and classification flow is represented in Figure 1.

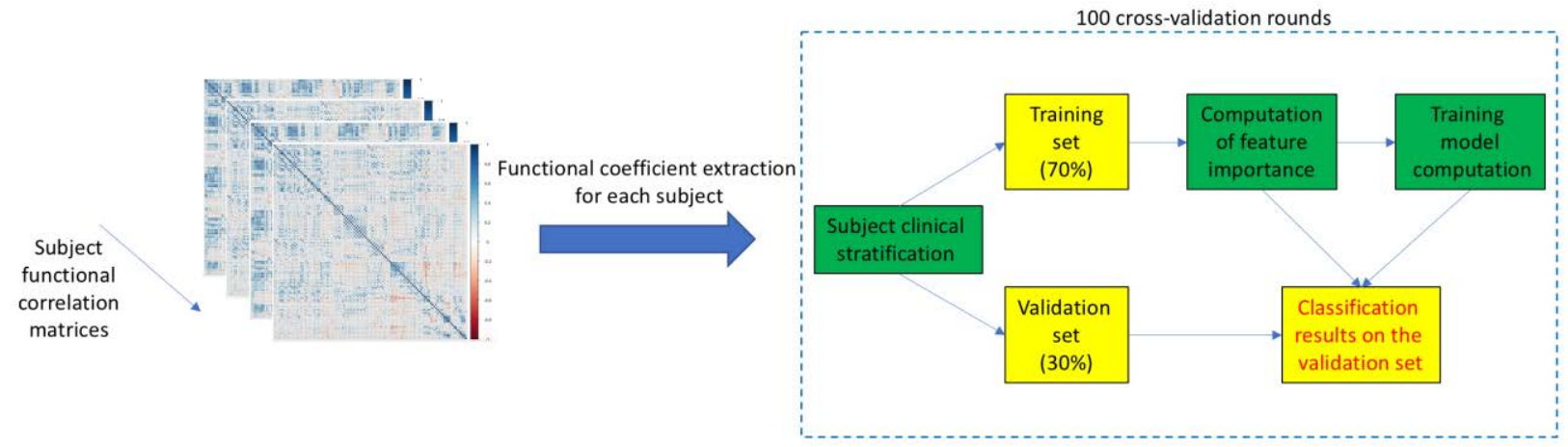

Figure 1: The classification process consists of different steps: (i) functional coefficients are extracted from each subject; (ii) The sample is stratified in order to have a balanced subject number for each clinical group for each cross-validation; (iii) Training (70\%) and validation (30\%) subjects are sampled; (iv) From the training set, important features are extracted and classification models are computed; (v) Classification is carried out on the validation test based on the training models. The group (seizure or non-seizure) classification was performed with different machine learning models (Random Forest, Support Vector Machine and Neural Network) and steps (ii), (iii), (iv) and (v) were carried out within 100 round of crossvalidation.

We expected that RF, due to its internal bagging, is the most appropriate model to produce robust results in this study, where the number of features outnumbers the sample size. We also compared RF classification performances of several state-of-the-art classifiers that have been used to predict seizure outcome in other studies, allowing us to assess the stability of classification performance independent of the machine learning model. SVM, given a feature representation of each sample, aims to find a separating hyperplane so the distance between the hyperplane and the closest training samples of each class is maximized. NN are organized into layers: input, hidden, and output. The input layer consists of a set of neurons representing the input features and the output layer responds to information learned over the input and hidden layers during the training to produce a result. RF, SVM and NN (with a 4 unit hidden layer) were implemented with the latest version of R packages: randomForest, e1071 and nnet.

\section{RESULTS}

FC correlation coefficients allowed us to distinguish TBI patients who went on to experience at least one seizure from seizure-free subjects with a mean accuracy of $0.691 \pm 0.036$ (sensitivity: $0.647 \pm 0.056$; specificity: $0.737 \pm 0.054$ ) over the 100 rounds of cross-validation. This result remains valid using different machine learning techniques, as shown in Table 2, where classification performances obtained with RF, SVM and NN are reported.

RF performs slightly better than SVM and NN in terms of accuracy and specificity, and NN reports the highest sensitivity. However, the table shows that no significant difference can be found between different machine learning models, demonstrating the stability of the results regardless of the classifier used.

Following the assessment of each classifier's performance, we examined which brain regions were selected as significant from the RF feature extraction. We found 66 important features to distinguish seizure outcome that represent 66 correlation values of FC between pairs of anatomical regions corresponding to segmentation defined by the AAL atlas. These pairs are shown in Figure 2. 
Table 2: A comparison between different machine learning models (Random Forest, Support Vector Machine and Neural Network). Performances are reported in terms of accuracy, sensitivity and specificity and the related standard deviations. The best performance for each metric is reported in bold.

\begin{tabular}{|l|l|l|l|}
\hline Machine Learning Model & Accuracy & Sensitivity & Specificity \\
\hline Random Forest & $\mathbf{0 . 6 9 1} \pm \mathbf{0 . 0 3 6}$ & $0.647 \pm 0.056$ & $\mathbf{0 . 7 3 7} \pm \mathbf{0 . 0 5 4}$ \\
\hline Support Vector Machine & $0.675 \pm 0.035$ & $0.632 \pm 0.054$ & $0.723 \pm 0.054$ \\
\hline Neural Network & $0.690 \pm 0.035$ & $\mathbf{0 . 6 6 7} \pm \mathbf{0 . 0 5 5}$ & $0.715 \pm 0.049$ \\
\hline
\end{tabular}
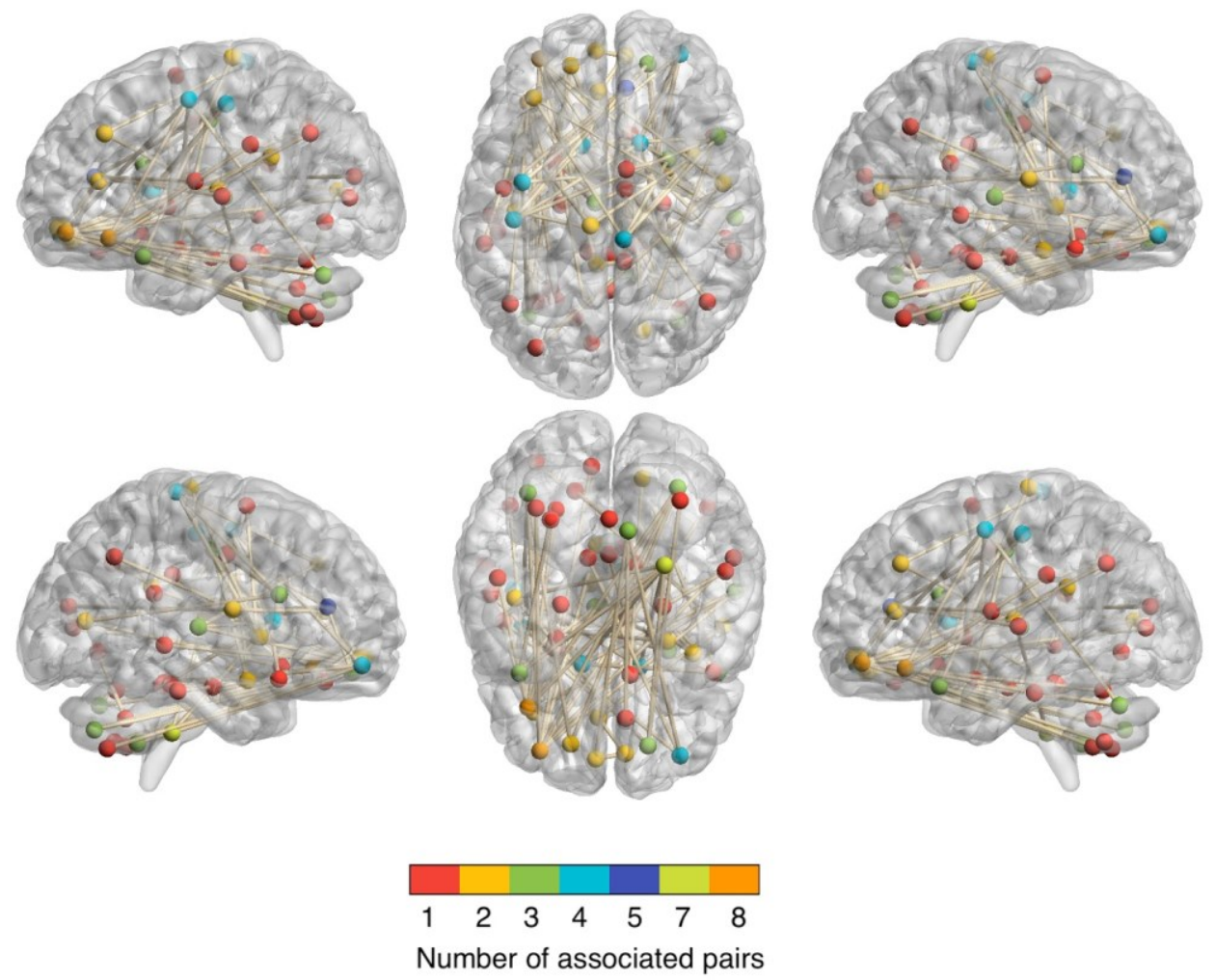

Figure 2: The brain connectivity graph (Xia, Wang, and He 2013) depicts 66 functional connections that were important to distinguish TBI patients who had at least 1 seizure from seizure-free subjects, according to the Random Forest classifier. Several regions were involved in multiple pairwise functional connections. The color of each node represents the number of significant connections the node formed. Six orientations of the brain are shown.

Within the 66 significant pairwise features identified, 60 unique regions of the 116 region AAL atlas were implicated. Several ROIs were contained in multiple pairs. Regions that were contained within more than three significant feature pairs are included in Table 3. 
Table 3: A list of ROIs involved in more than three features extracted from a machine learning model that best predict seizure susceptibility following TBI.

\begin{tabular}{|l|l|l|}
\hline $\begin{array}{c}\text { Region Number } \\
\text { (AAL Atlas) }\end{array}$ & \multicolumn{1}{|c|}{ Region Name } & \multicolumn{1}{|c|}{$\begin{array}{c}\text { Number of } \\
\text { Associated Pairs }\end{array}$} \\
\hline 1 & Left precentral gyrus & 4 \\
\hline 10 & Right middle frontal gyrus, orbital part & 4 \\
\hline 57 & Left postcentral gyrus & 4 \\
\hline 70 & Right paracentral lobule & 4 \\
\hline 71 & Left caudate nucleus & 4 \\
\hline 72 & Right caudate nucleus & 4 \\
\hline 32 & Right anterior cingulate gyrus & 5 \\
\hline 108 & Left lobule X of cerebellar hemisphere & 7 \\
\hline 9 & Left middle frontal gyrus, orbital part & 8 \\
\hline 15 & Left inferior frontal gyrus, pars orbitalis & 8 \\
\hline
\end{tabular}

\section{DISCUSSION}

\subsection{Discussion}

This study has focused on the feasibility of using fMRI to predict seizure outcome following TBI using several well-validated machine learning techniques. RF, SVM, and NN performed similarly, with no statistically significant differences between their accuracy, sensitivity, and specificity values.

Few studies have used imaging data to predict seizure onset in patient populations. The majority of studies have examined other data modalities (i.e. EEG) within epileptic cohorts that have specific forms of focal epilepsy characterized by a lower variability than PTE (Lai et al. 2017; Munsell et al. 2015). The results obtained in our study are especially promising if we consider that similar investigations have used larger study samples and EEG data, which are more sensitive to the seizure occurrence.

Our method did not obtain as high specificity or accuracy results compared with other studies that examined the predictive power of EEG to assess seizure-onset or epilepsy, although this may be attributable to the difference in quantity of data points acquired from fMRI versus EEG. For instance, our method resulted in slightly lower specificity than Kiral-Kornek et al.'s deep learning method for seizure prediction that examined over 16 years of intracranial EEG containing 2817 seizures from ten patients (Kiral-Kornek et al. 2018). The study did not indicate a sampling rate, although the sampling rate for intracranial EEG can be as high as $2000 \mathrm{~Hz}$ (Davis et al. 2018). Even considering a smaller sampling rate, this study would have significantly more data available for the training and validation phases. Additionally, literature on seizure prediction using SVM classifiers reported slightly higher specificity than any of our models, ranging from 3\%-5\% improvement (Teixeira et al. 2014; Rasekhi et al. 2013; Bandarabadi et al. 2015; Usman, Usman, and Fong 2017), although these studies also trained classifiers using EEG data that contained seizures within multiple EEG channels for up to 224 subjects (Teixeira et al. 2014).

In our study, 66 extracted predictive features elucidated several ROIs that may potentially be more influential regions because of their implication in multiple features. The extracted ROIs highlight a diverse community of regions across the brain, including anterior and posterior cortical regions as well as 
subcortical basal ganglia. Several regions, namely the left precentral gyrus, right paracentral lobule, right caudate nucleus, left caudate nucleus, and left lobule X of cerebellar hemisphere implicate a connection to motor action, innervation, and coordination (Afifi and Bergman 1998), suggesting there may be a connection between FC of motor-related regions and simple focal onset motor seizures. At the time of this study, seizure type was not available for all patients, as seizure/no-seizure classification was determined by binary survey questions that probed the occurrence of seizures within specified windows following injury. The additional regions identified in Table 3 - right middle frontal gyrus, left postcentral gyrus, right anterior cingulate gyrus, orbital part of the left middle frontal gyrus, and pars orbitalis of the left inferior frontal gyrus - represent regions associated with complex cognitive functions, including attention orientation and response inhibition, and primary somatosensory processing (Afifi and Bergman 1998). The ten regions found to be involved in the greatest number of pairwise functional connections were not hubs in the default mode network, the network most often cited in resting-state connectivity studies.

Several of the regions involved in more than 3 pairwise connections correspond with features found by Zhang et al. to be important to distinguish epileptic from healthy subjects based on fMRI data. The right caudate nucleus, which is involved in 4 features in our model, was in one of the top 20 links of their SVM classification. The precentral gyrus, postcentral gyrus, and orbital part of inferior frontal gyrus, associated with 4, 4, and 8 features in our model, were also obtained as significant regions, although in the opposite hemisphere as our findings (Zhang et al. 2012).

The three regions involved in the highest number of pairwise features were located in the left hemisphere, showing a potential hemispheric bias. In future investigation, we aim to reexamine these results taking into account the lesion location and region of seizure onset, as the unilateral result may have been biased due to either or both conditions.

\section{CONCLUSIONS AND FUTURE WORK}

Our machine learning model was able to successfully identify alterations in functional brain connectivity that most strongly predict seizure outcome following TBI. This study is the first to use machine learning to identify seizure outcome from a sample that consists only of patients. To the best of our knowledge, current studies have obtained better accuracy and sensitivity values only to predict seizures immediately prior to onset, or to discriminate epileptic subjects from healthy controls.

As presented, we investigated how accurately a machine learning model could predict seizure susceptibility based on seizures reported in the PTE Ottman and GOS-E questionnaires. As we gather more clinical data and fMRI results in upcoming years as the EpiBioS4Rx progresses and reaches its goal of 300 patients, we will reassess how RF, SVM, and NN classifiers may predict seizure outcome following TBI. We also aim to explore how resting state connectivity correlates with the latency until seizure onset. Incoming data will include more comprehensive longitudinal seizure reporting, as well as PTE diagnoses from clinical experts, allowing for a more nuanced application of our machine learning model.

Lastly, we plan to compare our model to one that introduces age-matched healthy controls from a publicly available cohort with a protocol similar to EpiBioS4Rx to expand the training set.

\section{ACKNOWLEDGMENTS}

This study was conducted with the support of the National Institute of Neurological Disorders and Stroke (NINDS) of the National Institutes of Health (NIH) under award number U54 NS100064 (EpiBioS4Rx).

\section{REFERENCES}

Afifi, Adel K, and Ronald A. Bergman. 1998. Functional Neuroanatomy: Text and Atlas. 2nd Editio. New York: McGraw-Hill, Health Professions Division. 
Amoroso, Nicola, Marianna La Rocca, Stefania Bruno, Tommaso Maggipinto, Alfonso Monaco, Roberto Bellotti, and Sabina Tangaro. 2018. "Multiplex Networks for Early Diagnosis of Alzheimer's Disease." Frontiers in Aging Neuroscience. https://doi.org/10.3389/fnagi.2018.00365.

Bandarabadi, Mojtaba, César A. Teixeira, Jalil Rasekhi, and António Dourado. 2015. "Epileptic Seizure Prediction Using Relative Spectral Power Features." Clinical Neurophysiology. https://doi.org/10.1016/j.clinph.2014.05.022.

Breiman, Leo. 2001. "Random Forrest." Machine Learning. https://doi.org/10.1023/A:1010933404324.

Davis, Kathryn A., Seth P. Devries, Abba Krieger, Temenuzhka Mihaylova, Daniela Minecan, Brian Litt, Joost B. Wagenaar, and William C. Stacey. 2018. "The Effect of Increased Intracranial EEG Sampling Rates in Clinical Practice." Clinical Neurophysiology. https://doi.org/10.1016/j.clinph.2017.10.039.

Fergus, P., A. Hussain, David Hignett, D. Al-Jumeily, Khaled Abdel-Aziz, and Hani Hamdan. 2016. "A Machine Learning System for Automated Whole-Brain Seizure Detection." Applied Computing and Informatics. https://doi.org/10.1016/j.aci.2015.01.001.

Frey, Lauren C. 2003. "Epidemiology of Posttraumatic Epilepsy: A Critical Review.” Epilepsia 44 (s10): $11-17$.

Friston, K. J., A. P. Holmes, K. J. Worsley, J. -P Poline, C. D. Frith, and R. S.J. Frackowiak. 1994. "Statistical Parametric Maps in Functional Imaging: A General Linear Approach." Human Brain Mapping. https://doi.org/10.1002/hbm.460020402.

Hillary, F. G., J. Slocomb, E. C. Hills, N. M. Fitzpatrick, J. D. Medaglia, J. Wang, D. C. Good, and G. R. Wylie. 2011. "Changes in Resting Connectivity during Recovery from Severe Traumatic Brain Injury." International Journal of Psychophysiology. https://doi.org/10.1016/j.ijpsycho.2011.03.011.

Hillary, Frank G., Sarah M. Rajtmajer, Cristina A. Roman, John D. Medaglia, Julia E. Slocomb-Dluzen, Vincent D. Calhoun, David C. Good, and Glenn R. Wylie. 2014. "The Rich Get Richer: Brain Injury Elicits Hyperconnectivity in Core Subnetworks." PLoS ONE. https://doi.org/10.1371/journal.pone.0104021.

Humphreys, Ioan, Rodger L. Wood, Ceri J. Phillips, and Steven Macey. 2013. "The Costs of Traumatic Brain Injury: A Literature Review." ClinicoEconomics and Outcomes Research. https://doi.org/10.2147/CEOR.S44625.

Immonen, Riikka, Irina Kharatishvili, Olli Gröhn, and Asla Pitkänen. 2013. "MRI Biomarkers for PostTraumatic Epileptogenesis." Journal of Neurotrauma $30 \quad$ (14): 1305-9. https://doi.org/10.1089/neu.2012.2815.

Iraji, Armin, Hanbo Chen, Natalie Wiseman, Robert D. Welch, Brian J. O’Neil, E. Mark Haacke, Tianming Liu, and Zhifeng Kou. 2016. "Compensation through Functional Hyperconnectivity: A Longitudinal Connectome Assessment of Mild Traumatic Brain Injury." Neural Plasticity. https://doi.org/10.1155/2016/4072402.

Karlık, Bekir, and Şengül Bayrak Hayta. 2014. "Comparison Machine Learning Algorithms for Recognition of Epileptic Seizures in EEG." In Proceedings IWBBIO 2014.

Kiral-Kornek, Isabell, Subhrajit Roy, Ewan Nurse, Benjamin Mashford, Philippa Karoly, Thomas Carroll, Daniel Payne, et al. 2018. "Epileptic Seizure Prediction Using Big Data and Deep Learning: Toward a Mobile System.” EBioMedicine. https://doi.org/10.1016/j.ebiom.2017.11.032.

Lai, Chunren, Shengwen Guo, Lina Cheng, and Wensheng Wang. 2017. "A Comparative Study of Feature Selection Methods for the Discriminative Analysis of Temporal Lobe Epilepsy." Frontiers in Neurology. https://doi.org/10.3389/fneur.2017.00633.

Messori, Anna, Gabriele Polonara, Flavia Carle, Rosaria Gesuita, and Ugo Salvolini. 2005. "Predicting Posttraumatic Epilepsy with MRI: Prospective Longitudinal Morphologic Study in Adults." Epilepsia $46(9)$. 
Mishra, Asht Mangal, Harrison Bai, Alexandra Gribizis, and Hal Blumenfeld. 2011. "Neuroimaging Biomarkers of Epileptogenesis." Neuroscience Letters. https://doi.org/10.1016/j.neulet.2011.01.076.

Mishra, Asht Mangal, Xiaoxiao Bai, Basavaraju G. Sanganahalli, Stephen G. Waxman, Olena Shatillo, Olli Grohn, Fahmeed Hyder, Asla Pitkänen, and Hal Blumenfeld. 2014. "Decreased Resting Functional Connectivity after Traumatic Brain Injury in the Rat." PLOS ONE. https://doi.org/10.1371/journal.pone.0095280.

Munsell, Brent C., Chong Yaw Wee, Simon S. Keller, Bernd Weber, Christian Elger, Laura Angelica Tomaz da Silva, Travis Nesland, Martin Styner, Dinggang Shen, and Leonardo Bonilha. 2015. "Evaluation of Machine Learning Algorithms for Treatment Outcome Prediction in Patients with Epilepsy Based on Structural Connectome Data." NeuroImage. https://doi.org/10.1016/j.neuroimage.2015.06.008.

Mursalin, Md, Yuan Zhang, Yuehui Chen, and Nitesh V. Chawla. 2017. "Automated Epileptic Seizure Detection Using Improved Correlation-Based Feature Selection with Random Forest Classifier." Neurocomputing. https://doi.org/10.1016/j.neucom.2017.02.053.

Obukhov, K., I. Kersher, I. Komoltsev, and Yu. Obukhov. 2018. "Epileptiform Activity Detection and Classification Algorithms of Rats with Post-Traumatic Epilepsy." Pattern Recognition and Image Analysis. https://doi.org/10.1134/S1054661818020153.

Pinto-Orellana, Marco A., and Fabio R. Cerqueira. 2017. "Patient-Specific Epilepsy Seizure Detection Using Random Forest Classification over One-Dimension Transformed EEG Data." In Advances in Intelligent Systems and Computing. https://doi.org/10.1007/978-3-319-53480-0_51.

Pippa, Evangelia, Vasileios G Kanas, Evangelia I Zacharaki, Vasiliki Tsirka, Michael Koutroumanidis, and Vasileios Megalooikonomou. 2017. "EEG-Based Classification of Epileptic and Non-Epileptic Events Using Multi-Array Decomposition." International Journal of Monitoring and Surveillance Technologies Research. https://doi.org/10.4018/IJMSTR.2016040101.

Rajpoot, Kashif, Atif Riaz, Waqas Majeed, and Nasir Rajpoot. 2015. "Functional Connectivity Alterations in Epilepsy from Resting-State Functional MRI." PLoS ONE. https://doi.org/10.1371/journal.pone.0134944.

Rasekhi, Jalil, Mohammad Reza Karami Mollaei, Mojtaba Bandarabadi, Cesar A. Teixeira, and Antonio Dourado. 2013. "Preprocessing Effects of 22 Linear Univariate Features on the Performance of Seizure Prediction Methods." Journal of Neuroscience Mods. https://doi.org/10.1016/j.jneumeth.2013.03.019.

Staba, Richard J, Matt Stead, and Gregory A Worrell. 2014. "Electrophysiological Biomarkers of Epilepsy." Neurotherapeutics: The Journal of the American Society for Experimental NeuroTherapeutics 11 (2): 334-46. https://doi.org/10.1007/s13311-014-0259-0.

Teixeira, C Alexandre, Bruno Direito, César Alexandre Teixeira, Mojtaba Bandarabadi, Michel Le Van Quyen, Mario Valderrama, Bjoern Schelter, et al. 2014. "Epileptic Seizure Predictors Based on Computational Intelligence Techniques: A Comparative Study with 278 Patients." Computer Methods and Programs in Biomedicine. https://doi.org/10.1016/j.cmpb.2014.02.007.

Usman, Syed Muhammad, Muhammad Usman, and Simon Fong. 2017. "Epileptic Seizures Prediction Using Machine Learning Methods." Computational and Mathematical Methods in Medicine. https://doi.org/10.1155/2017/9074759.

Xia, Mingrui, Jinhui Wang, and Yong He. 2013. "BrainNet Viewer: A Network Visualization Tool for Human Brain Connectomics." PLoS ONE. https://doi.org/10.1371/journal.pone.0068910.

Yan. 2010. "DPARSF: A MATLAB Toolbox for 'Pipeline' Data Analysis of Resting-State FMRI." Frontiers in System Neuroscience. https://doi.org/10.3389/fnsys.2010.00013.

Zhang, Jie, Wei Cheng, Zheng Ge Wang, Zhi Qiang Zhang, Wen Lian Lu, Guang Ming Lu, and Jianfeng Feng. 2012. "Pattern Classification of Large-Scale Functional Brain Networks: Identification of 
Garner, La Rocca, Barisano, Vespa, Toga and Duncan

Informative Neuroimaging Markers for Epilepsy." PLoS ONE. https://doi.org/10.1371/journal.pone.0036733.

\section{AUTHOR BIOGRAPHIES}

RACHAEL GARNER holds a B.A. in Cognitive Science from the University of California, Berkeley and is currently a Project Assistant at the Laboratory of Neuro Imaging at USC Stevens Neuroimaging and Informatics Institute. Her research interests include neurodegenerative disorders, epilepsy, and cognitive disorders. Her email address is rachael.garner@loni.usc.edu

MARIANNA LA ROCCA received her Ph.D. from Bari University. Currently, she is a Postdoctoral Scholar-Research Associate in the USC Stevens Neuroimaging and Informatics Institute at the University of Southern California. Her research interests include image processing, pattern recognition, machine learning techniques, and complex networks applied to neuroscience. Her email address is marianna.larocca@loni.usc.edu.

GIUSEPPE BARISANO received his M.D. from San Raffaele University. He is currently a Ph.D. student at the University of Southern California. His research interests include Alzheimer's and dementias, epilepsy, and neurooncology. His email address is giuseppe.barisano@loni.usc.edu.

PAUL VESPA is a Professor of Neurosurgery and Neurology at the UCLA School of Medicine. He holds an M.D. from Ohio State University. His research interests include traumatic brain injury, intracerebral hemorrhage, coma, seizures, brain imaging, and stroke. His email address is pvespa@mednet.ucla.edu.

ARTHUR W. TOGA received his Ph.D. from St. Louis University, and serves as the Director of Laboratory of Neuro Imaging and USC Stevens Institute of Neuroimaging and Informatics. His research interests include neuroimaging, informatics, mapping brain structure and function, and brain atlasing. His email address is toga@loni.usc.edu.

DOMINIQUE DUNCAN is an Assistant Professor of Neurology in the Laboratory of Neuro Imaging at the University of Southern California. She holds a Ph.D. in Electrical Engineering from Yale University. Her research interests include developing analytic tools to study epilepsy, particularly after traumatic brain injury, and combining machine learning and virtual reality for MRI segmentation. Her email address is dominique.duncan@loni.usc.edu. 